\title{
Sudden Hemiplegia by Epidural Hematoma in a Patient Treated with Warfarin and Therapeutic Range International Normalized Ratio
}

\author{
Hyeong-Won Seo, Hyung Wook Park, Ki Hong Lee, Joon-Ho Ahn, Nam Sik Yoon, Jeong Gwan Cho \\ Department of Internal Medicine, Chonnam National University Hospital, Gwangju, Korea
}

\begin{abstract}
Spontaneous spinal epidural hematoma is extremely rare. It can cause fatal complication such as permanent neurologic deficit. Even though the exact cause of epidural hematoma is not known in most cases, anticoagulation treatment is associated in some cases. In this situation, prompt reversal of anticoagulation and immediate decompression surgery is required. Seventy-year-old woman presesnted with sudden hemiplegia. She had history of hypertension, dyslipidemia, and paroxysmal atrial fibrillation and had taken antihypertensive agent, statin, and warfarin. She did not have any history of trauma and her international normalized ratio was within therapeutic range during follow-up and at the time of event. Physical examination revealed decreased right extremities' motor and sensory function but cognitive function was normal. Detailed neurologic examination and spinal magnetic resonance imaging identified extensive, spontaneous spinal epidural hematoma. After the reversal of anticoagulation with vitamin $\mathrm{K}$ injection, the patient underwent emergent hematoma removal surgery and recovered completely. Our case suggests epidural hematoma should be considered in sudden hemiplegia patients with anticoagulation therapy. Immediate reversal of anticoagulation and decompression surgery can avoid neurologic deficit.
\end{abstract}

Keywords: Hemiplegia; Epidural hematoma; Warfarin

\section{INTRODUCTION}

A spinal subdural or epidural hematoma may result from back trauma, anticoagulation or thrombolytic therapy, or, in patients with bleeding diathesis, lumbar puncture [1]. Around 15\% to $20 \%$ of epidural hematomas are fatal [2]. The most common cause of epidural hematoma is traumatic, although spontaneous hemorrhage is known to occur or as a rare complication of anesthesia (such as epidural anesthesia) or surgery (such as laminectomy). Oral anticoagulation therapy is recommended to prevent thromboembolic events in high risk patients with atrial fibrillation $(\mathrm{AF})$ [3]. Anticoagulation therapy with warfarin always has potential risk of various complications even in patients with therapeutic range of international normalized ratio (INR) [4]. We report sudden hemiplegia by extensive, spontaneous spinal epidural hematoma in an anticoagulated patient with therapeutic range INR.

\section{CASE REPORT}

A 70 year-old female was admitted to the emergency department with sudden weakness of right arm and leg. She had history of hypertension, dyslipidemia, and paroxysmal AF and had taken antihypertensive agent, statin, and warfarin for 6 years. Her vital sign was stable as such blood pressure $110 / 60 \mathrm{~mm} \mathrm{Hg}$, heart rate $86 / \mathrm{min}$, and body temperature $36.8^{\circ} \mathrm{C}$.

There was no abnormal finding on physical examination. However motor strength in right upper and lower extremities were reduced and pain, temperature sensations below $\mathrm{T} 2$ were decreased. Cognitive function, cranial nerve examination were normal. Laboratory results including coagulation profile did not show any remarkable abnormality (hemoglobin, $11.0 \mathrm{~g} / \mathrm{dL}$; fibrinogen, 285.5 $\mathrm{mg} / \mathrm{dL}$; fibrin degradation products, $2.7 \mu \mathrm{g} / \mathrm{mL}$; D-dimer, 0.58 $\mathrm{mg} / \mathrm{L}$; activated partial thromboplastin time, 55.2 seconds; and 
INR, 2.53). She had been treated in Chonnam National University Hospital for 6 years and INR value was within nearly therapeutic range, between two to three.

There was no abnormal image suggesting stroke events on prompt brain magnetic resonance imaging (MRI). Spinal cord MRI was done immediately after because we suspected cervical and thoracic spinal cord compression. It showed multiloculated cystic mass $(11 \times 1.5 \times 1 \mathrm{~cm})$ in spinal canal at posterior and right posterolateral region from $\mathrm{C} 1$ to $\mathrm{T} 2$ level with mass effect on spinal cord and compressive myelopathy at C6 to 7 at right side (Fig. 1). We suspected mostly hematoma because the patient's symptom developed suddenly without any history of trauma, bleeding dia-
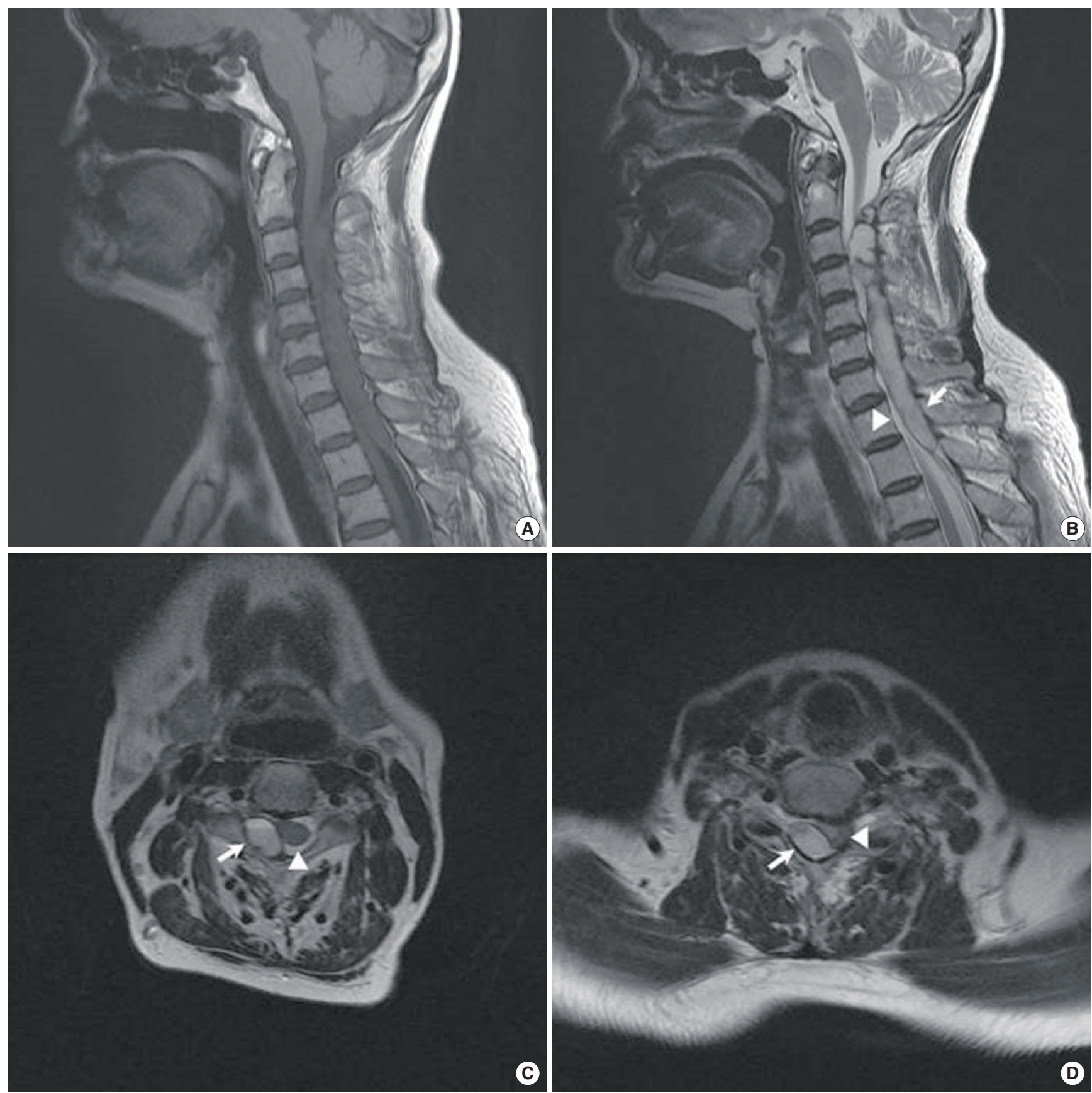

Fig. 1. Spinal epidural hematoma (white arrow) at posterior and right posterolateral region of C1 to T2 level as (A) low signal intensity in T1-weighted sagittal image and (B) high signal intensity in T2-weighted sagittal image. Spinal epidural hematoma compressed spinal cord (white arrow head) deviated to left side with compressive myelopathy at C6 to7 in T2-weighted axial image at (C) C3 level and (D) C6 level. 
thesis but she was on warfarin. The patient underwent emergency right cervical decompressive laminectomy and hematoma removal after intravenous administration of $5 \mathrm{mg}$ of vitamin K. Followup cervical MRI showed no remained hematoma in spinal canal. Strength of arm recovered completely and lower limb strength was restored with a power grade $4 / 5$ after rehabilitation. Even though there are few reports about the benefit of new oral anticoagulant in this situation, we used new oral anticoagulant after verification of resolving hematoma because it is known to reduce overall bleeding events compared to warfarin. The patients had been well for 8 months follow-up.

\section{DISCUSSION}

Spinal hematoma can lead to death or permanent neurologic deficit if it is left without prompt, adequate treatment. The incidence of spontaneous epidural hematoma is about one in one million individuals per year with a male preponderance of 3:1, occurring most commonly between the ages of 42 to 52 years [5]. Most spinal hematomas are located dorsally to the spinal cord in the cervicothoracic and thoracolumbar regions. The patients on anticoagulants are usually on drugs for other concurrent or primary illness. Spinal hematomas occurring during anticoagulant use are epidural or subdural in location. Intramedullary hematomas and subarachnoid spinal hemorrhage are also encountered during anticoagulant therapy though much rare. Dorsal and lumbar areas are most commonly involved on anticoagulant therapy but cervicothoracic area can also be involved like our case.

The spontaneous bleeding accounts for $0.3 \%$ to $0.9 \%$ of the total spinal epidural bleeds. This is associated with risk factors for coagulation abnormalities, platelet dysfunction like in polycythemia, soft tissue trauma, lumbar puncture, underlying neoplasm, conditions causing raised intravascular pressure (such as sneezing, lifting weights, pregnancy, and Valsalva maneuver) and conditions with certain vascular pathologies like spinal vascular malformations, vasculitis, or Paget's disease. No etiologic factor can be identified in about one-third of cases of spinal hematomas but it is occurring on anticoagulant therapy, are even more uncommon. The final common endpoint pathology is bleeding from the valveless epidural vein. In our patient there was no risk factor as described earlier except that she was on anticoagulant. The patient denied any history of trauma, acupuncture, bleeding diathesis so that we could not help suspecting anticoagulation therapy as a cause of hematoma [5-7].
Anticoagulation is the cornerstone in the treatment of AF especially in high risk patient. Vitamin K antagonist has been used as a primary agent for the most high risk patients. However, strict control of warfarin concentration in blood is required to obtain benefit and avoid dreadful complications (serious bleeding anywhere in whole body). Neurologic deficit in AF patients usually indicate thromboembolic or hemorrhagic events within the brain. However, careful physical and neurologic examinations are very important to differentiate cerebral or peripheral neurologic accident. Anticoagulant therapy alone probably does not trigger spinal hemorrhage. It is likely that there may be small internal vertebral venous plexus abnormality together with increased pressure in order to cause spinal hemorrhage. These two factors are thought to be the predisposing factors causing spinal hematoma in patients on anticoagulants. On suspicion of intraspinal hemorrhage, anticoagulation must be reversed immediately. Emergency laminectomy and decompression of the spinal cord appear mandatory if permanent neurologic sequelae are to be minimized, though rare cases with rapid spontaneous recovery have been reported [8]. Previous reports showed that spinal epidural hematoma was possible to occur in patients within the therapeutic range on warfarin treatment $[9,10]$. In the current case, laboratory finding also suggested INR level within therapeutic range, and she did not have any trauma, underlying coagulopathic disease. It might be explained that combined hypertension, and undetectable small vascular abnormality, anticoagulation may have influenced the epidural bleeding. Spinal epidural hematoma should be considered one of the causes of sudden hemiplegia besides of acute stroke when focal neurologic deficit was found in patients on warfarin medication. Also, detailed neurologic examination is mandatory to differentiate spinal epidural hematoma from stroke.

\section{REFERENCES}

1. Morales Ciancio RA, Drain O, Rillardon L, Guigui P. Acute spontaneous spinal epidural hematoma: an important differential diagnosis in patients under clopidogrel therapy. Spine J 2008;8:544-7.

2. Lu Z, Zhu G, Qiu Y, Cheng X. Minimally-invasive aspiration and drainage for management of traumatic epidural hematoma straddling transverse sinus. Neurol India 2013;61:111-6.

3. Camm AJ, Lip GY, De Caterina R, Savelieva I, Atar D, Hohnloser SH, et al. 2012 focused update of the ESC Guidelines for the management of atrial fibrillation: an update of the 2010 ESC Guidelines for the management of atrial fibrillation: developed with the special contribution of the European Heart Rhythm Association. Eur Heart J 2012;33:2719-47.

4. McManus DD, Rienstra M, Benjamin EJ. An update on the prognosis of 
patients with atrial fibrillation. Circulation 2012;126:e143-6.

5. Woon CY, Peng BC, Chen JL. Spontaneous spinal epidural haematomas and the prognostic implications of interval to surgical decompression: a report of two cases. J Orthop Surg (Hong Kong) 2009;17:216-9.

6. Sarubbo S, Garofano F, Maida G, Fainardi E, Granieri E, Cavallo MA. Spontaneous and idiopathic chronic spinal epidural hematoma: two case reports and review of the literature. Eur Spine J 2009;18:1055-61.

7. Cincu R, de Asis Lorente F, Rivero D, Eiras J, Ara JR. Spontaneous subdural hematoma of the thoracolumbar region with massive recurrent bleed.
Indian J Orthop 2009;43:412-5.

8. Sagar A, Hassan K. Drug interaction as cause of spontaneously resolving epidural spinal hematoma on warfarin therapy. J Neurosci Rural Pract 2010;1:39-42

9. Kirazli Y, Akkoc Y, Kanyilmaz S. Spinal epidural hematoma associated with oral anticoagulation therapy. Am J Phys Med Rehabil 2004;83:220-3.

10. Lederle FA, Cundy KV, Farinha P, McCormick DP. Spinal epidural hematoma associated with warfarin therapy. Am J Med 1996;100:237-8. 